\title{
Une pensée de l'altérité chez Martin Heidegger
}

Jean Gobert Tanoh

\section{OpenEdition \\ Journals}

\section{Édition électronique}

URL : https://journals.openedition.org/leportique/1433

DOI : $10.4000 /$ leportique. 1433

ISSN : $1777-5280$

\section{Éditeur}

Association "Les Amis du Portique"

\section{Référence électronique}

Jean Gobert Tanoh, « Une pensée de l'altérité chez Martin Heidegger », Le Portique [En ligne], ePortique, mis en ligne le 07 décembre 2007, consulté le 10 septembre 2022. URL : http:// journals.openedition.org/leportique/1433; DOI : https://doi.org/10.4000/leportique.1433

Ce document a été généré automatiquement le 10 septembre 2022.

Tous droits réservés 


\title{
Une pensée de l'altérité chez Martin Heidegger
}

\author{
Jean Gobert Tanoh
}

Les accointances de Heidegger avec le parti national-socialisme en Allemagne, rendent aujourd'hui plus controversé l'aiguillon originel de sa pensée, si bien que, de Être et temps jusqu'à Achèvement de la métaphysique et Poésie, certains y voient des préoccupations politiques en habits philosophiques. Pour eux, la pensée du plus grand philosophe du XXe siècle apparaît moins éclatante en sa teneur philosophique, en raison de sa participation supposée ou réelle au parti nazi d'Hitler, dont les pratiques ont entraîné l'extermination de 6 millions de Juifs, avec comme triste symbole le camp d'Auswitch. On peut, sans doute, comprendre, dans cette perspective la position suivante de Levinas : "Je sais que l'hommage que je rends à Sein und Zeit paraîtra pâle au disciple du grand philosophe. Mais je pense que c'est par Sein und Zeit que demeure valable l'ouvre ultérieure de Heidegger, qui ne m'a produit une impression comparable. Non pas, vous le pensez qu'elle soit insignifiante ; mais elle est beaucoup moins convaincante. ${ }^{1}$ Pour mieux se faire comprendre, Levinas ajoute ceci: «Je ne dis pas cela à cause des engagements politiques de Heidegger, pris quelques années après Sein und Zeit, bien que je n'aie oublié ces engagements et que Heidegger ne soit disculpé à mes yeux de sa participation au nationalsocialisme. $»^{2}$ Cette précision trahit bien Levinas, quant à sa volonté de ne pas lier l'œuvre du penseur à ses engagements politiques. Sa pensée ultérieure n'est -elle pas l'explicitation de son intuition originelle, à savoir la question de l'être dont Être et temps reste "un langage hésitant ? " . En vérité, pour Levinas, si l'œuvre du penseur est moins convaincante après Sein und Zeit, c'est en raison du fait que la question de l'altérité n'a plus été explicitement abordée par le penseur. Toute sa pensée semble tourner autour de " la pureté de l'être », laquelle constitue aux yeux de Levinas une idéologie de la pureté raciale, comme le souligne, insidieusement, E. Faye, pour qui, selon Heidegger «le principe de l'institution d'une sélection raciale est métaphysiquement nécessaire. $»^{4}$ 
La question, qui nous préoccupe ici, est moins de défendre Heidegger (puisque nous sommes très loin des faits et des lieux de ce problème) que voir, du point de vue strict de sa pensée, s'il y a véritablement des fondements philosophiques de l'idéologie nazie. Certainement pas! Dès lors, il s'agira de montrer que l'œuvre ultérieure du penseur n'est qu'une explicitation de la question de l'être, à laquelle est inhérente celle de l'altérité .Dans quel sens? Pour le comprendre, nous allons à partir de l'idée de l'altérité dans Être et temps et l'insistance sur la question de l'être, voir comment le rapport avec l'autre acquiert sens et consistance. D'où les axes suivants qui déterminent notre méditation: de l'être-au-monde du Dasein, l'être comme souci et la question de la technique comme celle de l'altérité.

I De l'être-au-monde du Dasein

Le projet philosophique de Heidegger est de poser à nouveaux frais la question de l'être, car dit -il: «la question de l'être est aujourd'hui tombée dans l'oubli. $»^{5}$ Cette oubliance de la question résulte de l'envahissante métaphysique de la subjectivité, qui depuis le cogito de Descartes a, d'une certaine manière, substitué à tout absolu, la raison. Désormais, seule la raison constitue l'unique référence à partir de laquelle toute connaissance doit pouvoir trouver son fondement. Autrement dit, vouloir penser un autre fondement en dehors de la raison, c'est immanquablement s'engouffrer dans des connaissances aux bases moins rassurantes, incapables de bâtir une historialité consistante de l'homme. Mais pour Heidegger, la prétention à l'absolutisation de la raison est un non-sens. En effet, la raison comme essence de l'homme, ne peut être érigée en fondement absolu, pour autant qu'elle-même appelle un véritable fondement. C'est pourquoi, «le dépassement de la métaphysique ne mérite d'être pensé que lorsqu'on pense à l'appropriation-qui-surmonte l'oubli de l'être. $»^{6}$

Dépasser la métaphysique moderne de la subjectivité, c'est absolument pour Heidegger une interrogation radicale sur le fondement du connaître de l'homme. Et, cette interrogation n'est pertinente que lorsqu'elle a pour objet l'être: «La question à poser est celle du sens de l'être. ${ }^{7}$ Dire les choses de cette manière voudrait simplement signifier que la question essentielle et importante, sans laquelle de toute évidence, aucune question ne peut avoir de sens, est bien celle du sens ou de la vérité de l'être. Car, c'est dans la mesure où, ce sens sera explicite pour l'homme que celui-ci, s'élèvera à la vérité des choses. Dès lors, la question première dans cette recherche du fondement des fondements, est celle de l'élément de base qui rend possible celle-ci. Cet élément de base est l'étant : "Dans la mesure où l'être constitue le questionné et où être veut dire l'être de l'étant, la question de l'être va avoir pour interrogé l'étant lui-même. ${ }^{8}$ Mais de quel étant s'agit-il ? Il ne s'agit visiblement pas de n'importe quel étant. Il ne s'agit pas de l'étant stylo avec lequel nous écrivons, mais d'un étant privilégié, parce qu'en entente avec l'être, c'est-à-dire l'homme, qui dans la terminologie de Heidegger, est le Dasein : "Le Dasein (l'homme) a ceci de propre qu'il n'a qu'à être pour que cet être qui est le sien lui soit découvert. L'entente de l'être est lui-même une détermination d'être du Dasein. Ce qui distingue ontiquement le Dasein, c'est qu'il est ontologique. "

Dans sa démarche, Heidegger va s'imposer une analytique du Dasein, pour mettre en évidence les éléments constitutifs de celui-ci, par lesquels naturellement, deviennent non seulement explicite l'identité ontologique du Dasein mais aussi, et surtout le sens de l'être, objet fondamental de son intuition philosophique. En d'autres termes, ce que signifie l'être, ne peut être véritablement compris qu'avec une connaissance exacte de l'ontologie du Dasein. Ainsi, avec les éléments de l'analytique 
du Dasein s'offre à nous l'opportunité d'une détermination profonde et pertinente de la vérité de l'être : "L'universalité du concept d'être n'a rien d'incompatible avec la "spécialité " de la recherche, c'est-à-dire que pénétrer jusqu'au à lui, la recherche aura à en passer par l'interprétation spéciale d'un étant déterminé, le Dasein, en quoi elle atteindra l'horizon de l'entente de l'être et sa possible explicitation. $»^{10}$

Dans cette perspective, un concept demeure fondamental, c'est le concept de l'être-au-monde. Comme le souligne si bien Heidegger en sous-titre du deuxième chapitre de la première partie de Être et temps : «L'être-au-monde en général comme constitution fondamentale du Dasein. $»^{11}$ En quoi l'être-au-monde est-il fondamental? C'est par cela que le sens du Dasein comme existant est à apprécier. Et la clarification de son entente avec l'être intègre cette détermination de l'être-au-monde. Aussi est-il nécessaire de s'approprier clairement celle-ci pour expliciter le sens de l'être. Sinon le risque d'une confusion reste grand. "Le Dasein est l'étant qui en son être se rapporte extensivement à cet être. Par là est indiqué le concept formel d'existence. Le Dasein existe. $»^{12}$ Le Dasein existe ne veut pas dire simplement qu'il a une réalité d'être dans le temps et dans l'espace, mais bien plus, celle-ci n'a de consistance que si elle se réalise pleinement dans son entente avec être.

L'être-au-monde comme constitution fondamentale du Dasein est caractérisé par trois types de relation : l'être-auprès, l'être-soi-même et l'être-avec. Dans le fond, ces trois types ne sont pas irréductibles, de telle sorte qu'il est impossible de concevoir l'un sans l'autre. Les traits d'union sont, dans ce sens, significatifs. L'un appelle les autres. Cependant à bien regarder de près, on se rend compte d'une chose : l'être-au-monde est essentiellement déterminé par l'être-avec. Nulle part ne le dit Heidegger. Mais posonsnous la question suivante : quel sens y a-t-il à penser les deux autres, si Heidegger n'a pas la conviction dominante que l'homme partage profondément son être avec son semblable? Qu'un 'homme soit seul au monde n'exige nullement une étude, de soimême et des choses. C'est la vie en société qui commande une connaissance authentique l'homme et des choses, afin de la porter à sa vérité. En effet, c'est parce que l'autre participe à l'expression de mon être, qu'il apparaitt nécessaire de me saisir et de saisir ma relation avec les choses avec clarté. Car, en vérité, le monde dans lequel je suis est un monde partagé : "La clarification de l'être-au-monde a montré qu'il n' " y a » pas d'emblée et que jamais non plus n'est en définitive pas davantage donné d'emblée un je isolé sans les autres. Toutefois si dans l'être-au-monde, il y a coexistence des "autres ", s'ils sont fois déjà là avec. $»^{13}$ De ce point de vue, les autres ne sont pas simplement des hommes que je rencontre, envers qui je dois manifester de l'indifférence voire du mépris; mais des Daseins-miens, pour autant que dans mon propre être, je partage avec eux les mêmes réalités ontologiques et ontiques. Et plus vrai, c'est à partir de ma rencontre avec l'autre que, d'une manière ou d'une autre, mon être se définit. "Sur la base de cet êtreau-monde affecté d'un avec le monde est chaque fois toujours déjà celui que je partage avec les autres .Le monde du Dasein est un monde commun. $»^{14}$ Un monde commun, pas au sens de ce qui serait pour tous, mais au sens de ce chaque Dasein expérimente profondément sa dépendance à l'autre. Une dépendance non-aliénante, plutôt libératrice, dans la mesure où habite en moi toujours un aller-vers. Le Dasein qui m'est mien ne se structure que dans cet aller-vers, c'est-à-dire dans la rencontre avec l'autre. Autrement dit, la rencontre avec l'autre, comme coexistence, est une détermination essentielle de la vérité du Dasein: «Le Dasein qui m'est propre, dans la mesure où il a l'être-avec comme structure essentielle, n'est qu'en tant que coexistence à la rencontre d'autrui »15. C'est pourquoi, souligne Heidegger que l'autre n'est pas un étant ayant le genre d'être d'un 
utile mais proprement le Dasein qui m'est mien. Dès lors, «cet étant n'est pas ce qui préoccupe, il se tient dans le souci mutuel. $»^{16}$ Se tenir dans le souci mutuel, c'est partager les mêmes les aspirations profondes qui définissent chaque Dasein. Ces aspirations s'originent dans l'exigence d'une substantielle historialité. Car nul doute qu'en chaque Dasein habite profondément, ce constant désir d'une présence dynamique dans l'histoire. Parce que partageant cela, les hommes ne peuvent que se tenir dans le souci mutuel. La compréhension profonde et claire des choses rend libre chaque homme ou Dasein à l'égard de l'autre. Toutefois, comment le Dasein peut-il parvenir à cette compréhension profonde et claire? Pour Heidegger, c'est à partir du souci véritable, auquel fait déjà signe le souci mutuel, que le Dasein parviendra à authentiquement se comprendre et comprendre les choses. Le souci véritable est celui de l'être. Et c'est bien en ce sens que se trouve subtilement la pensée de l'altérité chez Martin Heidegger.

\section{L'Être comme souci}

"De même qu'à la préoccupation en tant que matière de dévoiler l'utilisable, de appartient la discernation même le souci mutuel est guidé par l'égard et l'indulgence. $\gg^{17}$ Peuton trouver meilleures expressions pour la considération de l'autre que ces concepts d'égard et d'indulgence ? Sans doute non, car il n'y a de véritables rapports avec l'autre qu'à partir du moment où ces réalités deviennent des principes a priori. Ainsi si Heidegger écrit ces concepts en italique, c'est sans doute pour montrer que ceux-ci doivent être saisis d'une manière fondamentale, dans la perspective de l'ontologie fondamentale ; qu'ils ne reçoivent leur véritable sens moral qu'en les situant en celle-ci. Alors l'apriorité de ces concepts suppose qu'ils soient inscrits dans la constitution fondamentale de l'être-au-monde du Dasein, de telle sorte que celui-ci se libère des considérations particulières et négatives où, malheureusement et bien souvent, l'autre se trouve enfermé. Que le Dasein qui m'est mien, ait du respect pour l'autre et manifeste la tolérance à son endroit, participe du fait je partage indiscutablement mon être-au-monde avec lui. Et je n'accède à l'épanouissement de mon être qu'en intégrant l'autre.

La question qu'il convient de se poser est de savoir si l'homme de la modernité représente pleinement cette exigence de son être, envahi qu'il est par la consommation de l'étant. Aussi longtemps que l'homme sera soumis à la consommation de l'étant, aussi longtemps sera-t-il incapable de se comprendre authentiquement. Tel est, certainement, le présupposé essentiel qui meut le penser heideggérien. Si par la suite, Heidegger ne parle plus explicitement de cette considération de l'autre, c'est moins par changement de conviction que par le souci d'une réalité plus fondamentale, sans laquelle toute chose manquerait de consistance. Cette réalité est l'être. Si la question de l'être préoccupe Heidegger au point d'aiguillonner sa pensée de bout en bout, c'est parce que, sans une explicitation du sens de l'être, l'homme ne peut être: «Sans une ouverture de l'être nous ne pourrions d'aucune façon être "les hommes»." ${ }^{18} \mathrm{En}$ clair, l'homme ne pourrait pas, non seulement réaliser les déterminations essentielles de son être mais surtout ne pourrait, donner à sa vocation d'être dans l'entente de l'être, tout son sens. Or, qu'est ce que l'homme, s'il ne se connaît même pas, du point de vue de son ontologie et de sa mission, sachant bien que cette dernière est déterminée par la première. Rien, sinon un étant quelconque qu'on pourra à peine distinguer des autres étants. 
Pour Heidegger, il n'y a donc pas de discussion. Le plus important et le plus difficile, est la vérité de l'être qu'il faut constamment rendre claire; car les autres questions comme celle de l'altérité qui nous préoccupe ici, sont substantiellement liées à elle. En comprenant la vérité de l'être, nous nous disposons à mieux comprendre les autres questions, essentielles pour notre existence: «Le questionner est de façon authentique et juste la seule façon de reconnaitre pleinement ce qui, du haut de son rang suprême, tient notre être-là en puissance. ${ }^{19} \mathrm{Ce}$ qui signifie que toutes les préoccupations légitimes de l'homme doivent s'analyser et s'apprécier dans la perspective de la question de l'être. Autrement dit,rien de ce qui détermine notre existence,au sens courant du terme,ne peut être vrai ,qu'à condition de le situer dans les profondeurs abyssales de l'être,en explicitant constamment l'entente de l'homme avec l'être. Cela est une exigence pour laquelle nous est requise une attention soutenue ; car l'être,qui loin d'être absolument indéterminé,se détermine et se manifeste par la temporalité. D'où apparaît chez Heidegger, la consubstantialité de l'être et du temps. Le temps est l'autre nom de l'être comme se retirer (temps). Clairière de l'être: "Présence (être) appartient à la clairière du se retirer (temps) apporte avec elle la présence (être).» ${ }^{20}$ En vérité, le concept du temps chez Heidegger, n'est pas à prendre au sens ordinaire, c'est-à-dire comme suite des maintenants. Bien qu'il ne rejette pas cela, il se préoccupe, cependant, du sens ontologique de celui-ci. Sans ce sens, il serait impossible de s'approprier authentiquement le temps; et qu'en conséquence l'homme ne pourrait s'y tenir dans un rapport humanisant. Exerçant une tyrannie sur l'homme, c'est dans la mesure où le temps est véritablement approprié, que celui-ci peut atténuer celle-ci ; et même mieux faire du temps un allié dans le développement de son être et de sa société.

Le temps courant, pour ainsi dire, est la manifestation d'un temps plus originel, si bien qu'il n'est ni subjectif ni objectif, c'est-à-dire n'appartenant ni au sujet ni à l'objet : « "Le temps » n'est là-devant ni dans le «le sujet » ni dans l" " objet », ni « dedans », ni "dehors » et il " est » antérieur à toute subjectivité objectivité parce qu'il est la possibilité même de cette " antériorité».» ${ }^{21}$ En conséquence, ce que nous offre le temps, est appelé nécessairement à se comprendre par rapport au temps véritable, c'est-à-dire les choses $\mathrm{du}$ temps ne peuvent être comprises fondamentalement en dehors du sens originel du temps courant. Le qualificatif subjectif ou objectif qu'on attribue repose moins sur ce sens que l'expérience que l'homme a avec le temps. Or, en toute logique, celui-ci étant un étant du temps, ne peut fonder la vérité du temps, sauf qu'en étant à l'écoute de sa vérité originelle. En clair, pour que l'homme accède à la vérité du temps, et tout ce qui s'y manifeste, il doit aller au-delà son rapport immédiat avec le temps: «Le « temps officiel » se révèle être ce temps, temps "dans lequel» se rencontre l'utilisable et l'étant làdevant intérieurs au monde. Cela impose de le nommer intratemporain.(...) Interpréter l'intratemporanéité, c'est pénétrer d'un regard plus originel l'essence du temps officiel, ce qui permet en même temps d'en cerner l' "être ». "22

Autant que l'être-auprès du Dasein, c'est-à-dire préoccupé par l'utilisable (donc l'étant intratemporain), l'être-soi-même et l'être-avec du Dasein, déterminant la structure de son être-au-monde, doivent être élucidés et compris par une interprétation originelle du temps. Car l'homme est, avant tout, un étant du temps. Les autres et moi sommes du temps et non simplement dans le temps. Parvenir donc à la compréhension de l'homme et de son rapport avec les autres, exige que nous demeurions dans une constante écoute du temps, l'autre nom de l'être. En définitive, nous devons aller à l'être pour une connaissance authentique de l'homme et son 
rapport avec l'autre. Cela voudrait dire que nous saisissions le sens de l'ek-sistence qui est un concept spécifique à Heidegger. Avec l'isolement du ek, le Dasein, dans une correspondance extatique parvient à la clairière de l'être, où se trouve la vérité fondamentale de toute chose, y compris la sienne. Dans la clairière de l'être, le Dasein se découvre, et fait l'expérience du souci mutuel comme expression du souci véritable. Par là, la vérité du Dasein qui m'est mien, se révèle être celle de l'autre. L'autre qui est aussi Dasein rejoint le Dasein qui m'est mien dans l'unique vérité de leur être, de telle sorte qu'en celle-ci ,le rapport Dasein-Dasein, ne soit plus celui d'une opposition, mais d'une union élevante, où chaque Dasein s'élève à la plénitude de son être dans sa particularité. C'est dans les profondeurs de leur unique vérité, comme souci de l'être, que se manifeste davantage la splendeur infinie de leurs particularités complémentaires. Alors, le plus essentiel, c'est la saisie constante et le demeurer permanent du Dasein dans cette unique.

Nous le constatons. Cette façon d'écrire l'ek-sistence diffère de la manière courante. Pendant que cette manière s'intéresse davantage au vécu quotidien de l'homme, l'ek-sistence dépasse le plan ontique pour se situer au plan de l'ontologie fondamentale. Heidegger reste fidèle à son intuition et à sa démarche. Ce n'est pas parce qu'il s'en est désintéressé ,mais bien au contraire, c'est pour mieux le définir et le maintenir dans l'éclat de sa vérité que Heidegger s'est résolu à penser l'ontologie fondamentale. C'est une exigence de notre être et une nécessité absolue pour notre époque, où règne la domination de l'étant ou du réel, consacrant ainsi le total oubli de l'être : «La primauté du réel travaille à l'oubli de l'être. Par cette primauté le rapport spécifique à l'être se trouve enseveli, qu'il convient de rechercher dans la pensée justement conçue. ${{ }^{23}}^{\mathrm{Ce}}$ rapport spécifique à l'être est ce qui importe pour Heidegger. Sans ce rapport, manque à l'homme la consistance ontologique et ontique. Dans ce cas, nous ne devons pas être surpris de la fébrilité notre société et de la banalisation de l'autre. Reprendre ce rapport afin que l'homme ait de la consistance, et représente d'une manière essentielle son rapport aux choses et surtout à l'autre, constitue le projet fondamental de Heidegger. Pour lui, l'homme ne doit pas perdre de vue sa dépendance à l'être, pour autant que, c'est en celle-ci qu'il expérimente le sens profond de son être, lequel est défini par l'ek-sistence. C'est un concept fédérateur, qui nous permet de comprendre les objectifs et les implications de toute l'analytique du Dasein faite dans Être et temps, qui, du reste, demeure l'œuvre majeure du penseur. L'ek-sistence apparaît, précisément, être le concept qui rend mieux le rapport spécifique de l'homme à l'être. Il met en évidence l'exigence pour l'homme de se tenir dans l'être et pour l'être. En d'autres termes, il s'agit de comprendre à nouveau que l'être doit être un souci pour l'homme. Le souci dont parle Heidegger n'a rien d'un tourment;il est,en effet, la sauvegarde permanente de ce qui est devenu certitude. L'être est devenu certitude .Dans cette perspective, la vocation de l'homme est d'assurer sa garde. Et, c'est par l'ek-sistence que devient manifeste cette vocation pour la vérité de l'être. "L'ek-sistence, pensée de façon extatique, ne coincide pas, ni dans son contenu, ni dans sa forme avec l'existentia. Dans son contenu, l'ek-sistence signifie ex-tase en vue de la vérité de l'être. " ${ }^{24} \mathrm{Par}$ là, il faut noter que de l'ek-sistence est à la fois vérité de l'être et vérité du Dasein ou de l'homme. Objectivement si Heidegger s'appesantit sur la vérité de l'être, c'est pour penser radicalement l'homme, afin l'établir sur un ferme fondement ontologique, sur lequel il pourra entreprendre avec assurance son devenir historial, en préservant ce le distingue essentiellement des autres étants, c'est-à-dire la pensée. Ek-sister signifie donc penser radicalement l'être. Ou plus exactement se laisser revendiquer par l'être 
afin qu'advienne autant sa vérité que la nôtre. Mais à proprement parler, il y a ni d'une part la vérité de l'être ni d'autre part la vérité du Dasein. La vérité est la vérité de l'être sans laquelle il n'y a pas de Dasein. Dès lors, c'est autant absurde qu'injuste de penser les préoccupations et les exigences quotidiennes de l'homme en dehors de la pensée de l'être. C'est à partir de celle-ci que nous recevons la lumière de leur compréhension et leur consistante représentation. Parmi ces préoccupations et exigences, nous avons le rapport avec l'autre, qui suppose au préalable une évaluation de ce qu'il est. Autrement dit, selon que l'autre vaut ou pas, en fonction de critères subjectifs, nous définissons notre rapport avec lui. Or, il ne devrait pas en être ainsi. A partir du moment où l'autre est un autre moi, il vaut déjà sans autre forme de considération. Il partage avec moi la même identité essentielle. Mais comment pouvons-nous parvenir à garder constamment cela afin d'humaniser nos rapports ? C'est en ek-sistant, c'est-à-dire en pensant et en demeurant dans l'être. Tel est absolument le sens de Lettre sur l'humanisme.

Aucune valeur, et particulièrement celle de mon rapport à l'autre ne peut être consistante sans un rapport ferme à l'être. «Ainsi l'humanisme consiste en ceci : réfléchir et veiller à ce que l'homme soit humain et non in-humain, "barbare ", c'est-à-dire hors de son essence. $\|^{25}$ Veiller à ce que l'homme soit humain, en demeurant dans son essence, c'est le conduire à représenter avec rigueur sa dépendance à l'être. En cette dépendance où, il est chez soi, l'homme expérimente dans la clarté les exigences de son vécu quotidien où, il découvre celle l'intersubjectivité. L'autre, comme identique sujet, impose nécessairement au je, sous la dictée de l'être, les valeurs essentielles qui anoblissent les rapports humains. Parmi celles-ci, Heidegger a relevé deux, auxquelles nous avons déjà fait allusion au début de ce deuxième point à savoir: l'égard et l'indulgence. Pour Heidegger, c'est incontestablement la prise en garde sérieuse de l'être qui donne du sens et de la valeur à tout ce importe pour l'homme. Celui-ci n'est humain que s'il est capable de se donner de la consistance, pour résister à la tentation de l'inhumain ; car, en effet, habitent en l'homme à la fois l'humain et l'inhumain. La force de dominer l'inhumain réside dans l'entente de l'homme avec l'être. C'est pourquoi, chez Heidegger, apparaît une mystique de la parole. Celle-ci est, n'en point douter, un élément déterminant les rapports humains. Elle peut être constructive ou déconstructive voire destructrice.

La parole n'est pas un simple instrument de communication; elle est proprement le signe du mystère de l'homme. Avec elle l'homme donne sens aux choses, en vue de pouvoir se déterminer par rapport à elles. La qualité et la pertinence de cette détermination dépendent, en toute logique, de ce sens; qui à son tour dépend de la parole. Nous constatons là l'importance fondamentale de la parole pour nous-mêmes, pour les autres et pour le monde. Si l'homme ne parlait pas, il n'aurait sans doute pas de barbarie dans le monde. Parler suppose donc que nous saisissions la parole dans sa vérité fondamentale, afin qu'elle nous élève à l'humanité, en structurant harmonieusement le rapport à l'autre. La parole parle d'une manière essentielle, c'està-dire en laissant être l'essence des choses, car elle " est la venue à la fois éclaircissante et célante de l'être lui-même. ${ }^{26} \mathrm{La}$ parole parle déjà ; c'est un parlé déjà parlé. C'est pourquoi « la parole est parlante ${ }^{27}$ C'est donc parce que la parole parle, que l'homme parle. La parole n'appartient pas en propre à l'homme .Et son parler ne peut être vrai qu'à condition qu'il corresponde au parlé déjà parlé parlant. La parole est une invite à demeurer dans l'entente avec l'être, pour que nous établissions un rapport authentique avec les autres et les choses. On ne peut donner à l'autre toute sa valeur qu'en 
établissant avec lui une relation, où la parole a pour souci unique, le vrai pour tous, où est préservée pour chaque homme toute sa dignité. Il apparait alors absurde que par la parole nous détruisions les autres, en formant des cloisonnements culturels, sociaux et raciaux exclusifs et oppressants. saisir l'universalité du genre humain en ce qui concerne son identité essentielle. C'est vraisemblablement à partir d'une existence inauthentique, c'est-à-dire en dehors de la garde de l'être, comme sa vocation, que le Dasein peut concevoir que le morceau de cire a plus de réalité que l'idée d'étendue. Autrement dit, l'homme ne s'enferme dans les apparences, en établissant des cloisonnements, quand il a seulement en vue le posé-là. Comment alors pourra t-il voir au-delà, dans l'autre, l'unique réalité qui les détermine? Sans doute non ! Or en demeurant dans l'être, l'homme parvient et affirme son identité commune avec l'autre. Ainsi, ils ne peuvent que se tenir dans l'harmonie et dans le respect mutuel ; sachant bien que toute atteinte à la dignité de l'autre, est atteinte à ma propre dignité. Dans cette perspective, la question de l'altérité chez Heidegger, dépasse largement le cadre l'individu pour prendre en compte son environnement. C'est, sans doute, pourquoi, Heidegger s'inquiète de la domination de la technique sur le monde.

III La question de la technique comme question de l'altérité

"L'âge atomique, entendu comme époque planétaire de l'humanité, est caractérisé par ce fait que la puissance du très puissant principium reddendoe rationis se déploie, d'une façon troublante et dépaysante, pour ne pas dire qu'elle se déchaine dans le domaine déterminant de l'existence humaine. $\aleph^{28} \mathrm{Il}$ apparaît clairement que, la domination de la terre par la technique, est une menace pour l'homme. En utilisant les mots: troublante et dépaysante, Heidegger montre que la menace est sérieuse; tant il est question de l'assise de l'homme. D'où la précision suivante : «Les mots « troublante et dépaysante » qui sont employés ici, ne sont pas pris dans un sens sentimental. Ils doivent être entendus à la lettre, en ce que le déchainement, unique en son genre, de l'appel à fournir raison menace tout ce qui pour l'homme constitue « son pays natal » et qu'il lui enlève tout sol et tout terrain permettant un enracinement, c'est-à-dire cet attachement au terroir dont jusqu'à présent sont sortis toute grande époque de l'humanité, tout esprit découvreur d'horizons, tout style donné aux formes humaines. $\rangle^{29}$ De quel pays natal est-il question? A coup sûr, il ne s'agit pas d'un espace géographique ; mais bien de l'entente de l'homme avec l'être. En effet, la métaphysique moderne de la subjectivité, qui a pour résultantes la science et la technique modernes, a absolutisé le rationnellement connaissable, si bien que seul l'étant représentable rationnellement est à prendre en compte. Ce qui veut dire qu'une chose n'a d'être que lorsqu'elle peut être représentée par la raison. Tout ce qui tombe en dehors de la représentation ne peut être digne de connaissance. La science et la technique modernes manifestent hautement cette exigence de la métaphysique moderne, de telle sorte qu'elles se présentent comme les normes véritables du connaître de l'homme. Seul le positif compte et rien d'autre. La science ne veut rien savoir d'autre, sauf ce qui est. .Cette visée détermine autrement le sens de la science; elle « devient donc recherche par le projet qui s'assure lui-même dans la rigueur de l'investigation. $»^{30}$ Cette rigueur a pour but de mettre en demeure l'étant de livrer tous les principes qui le déterminent, afin de mieux l'approprier. En orientant les choses de cette façon, l'homme moderne avoue que, ce qui est fondamental pour lui, est son rapport à l'étant. Le puissant principe de raison se présente, désormais, comme principe régulateur du rapport, tant avec lui- 
même qu'avec les choses. Il y a là un nivellement de tout, y compris l'homme. Ce nivellement atteste de la domination tyrannique de la science et la technique modernes sur l'homme. Nous pouvons comprendre mieux, à présent, les sens des «troublante et dépaysante.» L'homme perd, pour ainsi dire, toute lisibilité véritable de son être et sa présence au monde ; car n'étant pas plus dans son " pays natal ", il ne peut contempler la lumière au cœur des choses. Le sol sur lequel il marche se dérobe constamment à ses pieds. D'ailleurs comment pourrait-il être autrement puisqu'il est coupé de son assise ? Pour être sur la terre, l'homme quoiqu'il fasse ne doit s'écarter des exigences de sa vocation, celle d'assurer la garde de l'être.

Si l'homme n'a plus la vraie lisibilité de son être, comment pourra-t-il établir avec l'autre une relation humanisante. Absolument pas! En effet, je ne peux être avec l'autre dans un rapport humain que si je réalise mon appartenance essentielle commune avec l'autre. N'ayant plus de repères et de lumière véritable, parce que subjugué par la consommation de la technique, comment pourrai-je voir en l'autre toute la valeur qu'il incarne? Arrachée à son terroir, l'époque de l'homme moderne ne peut plus être celle de tout esprit découvreur d'horizons, de tout style donné aux formes humaines. Car l'attachement au terroir est la profondeur où l'homme se teint et est tenu depuis toujours. Cette profondeur est l'être, en qui l'homme trouve son être. Et c'est dans une relation substantielle avec l'être qu'il s'élève à la plénitude de son humanité. Les formes humaines dont parle Heidegger sont à entendre dans ce sens de la plénitude, où l'homme ne réalise pas simplement des formes, mais des vérités fondamentales qui doivent baliser son être et son agir. L'agir est toujours en vue de soi-même et des autres. Il n'a de sens que par rapport à cela, si bien c'est inconcevable de penser un seul instant qu'un acte poser est sans conséquence. De ce fait, il convient d'articuler rigoureusement l'être et l'agir, si tant est que l'agir est véritablement déterminé par l'être. Ce qui suppose alors une profonde saisie de l'être de l'homme.

Par là, doit être ramenée toute chose à l'être, c'est-à-dire toute chose doit être comprise dans la perspective l'exigence de l'appartenance de l'homme à l'être. Avec l'avènement de la technique, cela est devenu incontournable. En se préoccupant de la technique, Heidegger veut la saisir dans son essence afin de resituer le rapport de l'homme à elle. Quoique nécessaire la technique appelle de sérieuses questions maintenant pour que cette nécessité se manifeste dans sa juste mesure, en libérant en l'homme toutes les potentialités valorisantes de son existence. Une meilleure compréhension de l'essence de la technique est, pour Heidegger, le gage d'une meilleure utilisation de celle-ci. Au lieu de l'aliéner et de lui enlever toutes ses valeurs fondamentales, la technique participera au développement de l'existence authentique de l'homme. "Nous questionnons au sujet de la technique et rapport à elle Le rapport est libre, quand il ouvre voudrions ainsi préparer un libre notre être (Dasein) à l'essence (Wessen) de la technique $»^{31}$. Autrement dit, le pouvoir actuel accordé à la technique résulte d'une ignorance de son essence. En toute logique, l'ignorance de la vérité d'une chose conduit à un mauvais rapport avec elle. Si donc l'humanité actuelle est sous la consommation tyrannique de la technique, c'est sans parce qu'elle pense au seulement technique de la technique. L'essence de la technique est, pour elle, technique. Tel n'est pas le cas; elle "n'est absolument rien de technique. $\|^{32}$ L'essence de la technique est définie par l'être, en tant que mode de dévoilement de celui-ci. «La technique déploie son être dans la région où le dévoilement a lieu. $»^{33}$ Pour mieux se faire comprendre, Heidegger s'interroge : «; Où et comment a lieu le dévoilement s'il n'est pas le simple fait de l'homme? $»^{34} \mathrm{Il}$ répond : «Nous n'avons pas à aller chercher bien loin .Il est seulement nécessaire de percevoir 
sans prévention ce qui a toujours réclamé l'homme dans une parole à lui adressée, et cela d'une façon si décidée qu'il ne peut jamais être, si ce n'est comme celui auquel une telle parole s'adresse. $\|^{35}$ Qui est celui qui adresse la parole à l'homme? C'est bien l'être par qui et pour qui l'homme pense: «La pensée, obéissant à la voix de l'être cherche pour celui -ci la parole à partir de laquelle la vérité de l'être vient au langage. $»^{36}$

Dans ce cas, le lieu commun de l'essence de l'homme et de la technique, en tant que leur vérité fondamentale, est l'être. En y demeurant, l'homme réalise qu'il est le producteur de la technique, et tant que tel ,il ne doit nullement se laisser dominer par sa propre production. En cela, il s'arrache à la tyrannie de la technique, en manifestant ce que Heidegger appelle la sérénité, c'est-à-dire l'égalité d'âme devant toute chose, où l'homme ne perd pas de vue sa dépendance essentielle à l'être. Elle est, pour lui, la seule possibilité pouvant permettre de retrouver un nouvel enracinement. La sérénité "devant les choses et l'esprit ouvert au secret nous dévoilent la perspective d'un futur enracinement. Il pourrait même arriver que ce dernier fût un jour assez fort pour rappeler à nous, sous une forme nouvelle, l'ancien enracinement qui l'heure disparait si vite. $»^{37}$

Le nouvel enracinement n'est pas, pour Heidegger, une simple réalité du temps ou nouvel véritablement, mais l'enracinement, qui a de tout temps été, et qui malheureusement a perdu toute force aux yeux l'homme, où cependant celui-ci doit pouvoir se maintenir. Se maintenir dans cet enracinement, c'est pour l'homme avoir le souci de l'être, en étant profondément son berger. Et à cette seule condition qu'il parviendra à la substantialité de ses rapports. Etre le berger de l'être n'a rien d'une abstraction, c'est plutôt le nécessaire besoin de l'homme, où il donne au souci mutuel ,dans son sens existentiel, toute son effectivité ,en mettant à l'abri le monde de ce constat que fait Heidegger: "Sur la terre pro-vient un obscurcissement du monde. Les éléments essentiels de cet obscurcissement sont : la fuite des dieux, la destruction de la terre, la grégarisation de l'homme, la prépondérance du médiocre. $»^{38}$ Mais précise $\mathrm{t}-\mathrm{il}$ : «Le monde est toujours le monde de l'esprit. $»^{39}$ En vérité peut-on séparer le monde de l'esprit du monde comme univers existentiel de l'homme? Sans doute non, c'est pourquoi, « la décomposition $»^{40}$ de l'esprit affecte absolument l'univers existentiel de l'homme. l'admirable progrès de la science et la technique. D'où le reproche suivant que Heidegger adresse à l'Europe :

« L'Europe, qui dans un incurable aveuglement, se trouve toujours sur le point de se poignarder elle-même prise aujourd'hui dans un étau entre d'une part la Russie et l'Amérique d'autre part. La Russie et l'Amérique sont toutes deux au point de vue de la métaphysique la même chose; la même frénésie sinistre de la technique déchaînée, et de l'organisation sans racine de l'homme. $»^{41}$

Se réapproprier la relation avec l'être, constitue une nécessité par laquelle l'homme moderne peut éviter la décomposition de son monde, en sachant que celle-ci intègre les données essentielles de son rapport à l'autre et au monde. Il s'agit, pour lui, de donner un contenu humanisant à l'histoire : "L'homme historial pense, et quand il pense véritablement, c'est-à-dire dans la mesure où il est lui-même le lieu où s'approprie un destin, il pense en ayant rapport à l'histoire. ${ }^{42}$ Destin et histoire expriment l'appartenance de l'homme à l'être, si tant est que l'être destine l'homme à assurer sa garde. Or, il est évident, selon Heidegger, que l'histoire n'est rien d'autre que dévoilement de l'être. Dans ce cas, avoir pour destin la garde de l'être veut dire, précisément, porter à l'éclat 
l'histoire. Autrement dit ,l'histoire n'aura tout son que lorsque l'homme l'accomplira dans la perspective du dévoilement de l'être ;lequel exige une sérénité devant les produits de la techniques, qui sont d'ailleurs manifestation de celui-ci , si bien sûr, nous partons du principe que l'essence de la technique se trouve dans l'être. Cette vocation ne saurait, alors, s'exprimer au détriment de l'homme dans son rapport avec son alter ego, c'est-à-dire son semblable.

Conclusion

«Plein de mérite, c'est pourtant poétiquement que l'homme habite la terre $»^{43} \mathrm{Ce}$ vers de Holderlin que cite Heidegger exprime l'exigence d'une relation essentielle que l'homme ne saurait écarter, sous prétexte,d'être capable de se donner les moyens d'une existence authentique, sans recours à autre chose. Habiter la terre transcende le simplement historique pour s'enraciner dans l'anhistorique, là où toute chose se révèle dans sa splendeur première, afin qu'ainsi se dégage un rapport valorisant l'homme. Et c'est dans le sens de l'anhistorique comme relation essentielle, que se perçoit et se fonde la relation humanisante avec l'autre. Cet anhistorique est la Libre-Etendue, c'està-dire l'être où les choses apparaissent dans leur vérité. D'où la nécessité de se maintenir dans cette Libre-Etendue en demeurant sereins face à la technique, pour qu'advienne un monde sans obscurcissement, où l'autre demeure une valeur interchangeable comme "idole (qui) atteste en effet le divin. " ${ }^{44}$ Le monde deviendra, alors, la patrie comme "communauté d'hommes de raison $»^{45}$, pour autant que celle-ci marque distinctivement notre appartenance essentielle à l'être.

\section{BIBLIOGRAPHIE}

E. Faye, Heidegger, l'introduction du nazisme dans la philosophie. Autour des séminaires inédits de 1933-1935, Paris, Albin Michel, 2005

M. Heidegger, Achèvement de la poésie, trad. A. Froidecourt, Paris, Gallimard, 2005.

M. Heidegger, Acheminement vers la parole, trad. J. Beaufret, Paris, Gallimard, 1994.

M. Heidegger, Approche de Hölderlin, trad. J. Launay, Paris, Gallimard, 1962.-

M. Heidegger, Chemins qui ne mènent nulle part, trad. W. Brokmeir, Paris, Gallimard, 1962.

M. Heidegger, Essais et Conférences, trad. A. Préau, Paris, Gallimard, 1958.

M. Heidegger, Etre et Temps, trad : F. Vezin, Paris, Gallimard, 1986.

M. Heidegger, Introduction à la métaphysique, trad : G. Kahn, Paris, Gallimard, 1967.

M. Heidegger, Le principe de raison, trad : A. Préau, Paris, Gallimard, 1983.

M. Heidegger, Lettre à Richardson in Questions IV, trad .C. Röels, Paris, Gallimard, 1976.

M. Heidegger, Lettre sur l'humanisme in Questions III , trad. R. Munier, Paris, Gallimard, 1966.

E. Levinas, Ethique et infini, Paris, Fayard, 1982

J.-L. Marion, Dieu sans l'être, Paris, P.U.F, 2002 
P. Ouattara, Quel chemin vers une patrie en Afrique?, Abidjan, UCAO, 2006.- préface du Pr A. Dibi

\section{NOTES}

1. Emmanuel Levinas, Ethique et Infini, Paris, Fayard, 1982.- p.38.

2. Ibid.

3. Martin Heidegger, Le principe de raison, Paris, Gallimard,1983, p.192.- trad. A. Préau

4. Emmanuel Faye, Heidegger, l'introduction du nazisme dans la philosophie. Autour des séminaires inédits 1933-1935, Paris, Albin Michel, 2005.- p.395

5.Martin Heidegger, Etre et Temps, Paris, Gallimard, 1986, p.25.- trad. F. Vezin

6. Martin Heidegger, Essais et conférences, Paris, Gallimard, 1958, p.90.- trad. A. Préau

7. Martin Heidegger, Etre et Temps, p.28.

8. Ibid, p.30.

9. Ibid, p.36.

10. Ibid, p.67.

11. Ibid, p.86.

12. Ibid, p.86.

13. Ibid, p.158.

14. Ibid, p.160.

15. Ibid, p.163.

16. Ibid, p.163.

17. Ibid, p.165.

18. Martin Heidegger, Introduction à la philosophie, Paris, Gallimard, 1967, p.91.- trad.G.

Kahn

19. Ibid, p.92.

20. Martin Heidegger, Lettre à Richardson in Questions IV, Paris, Gallimard, 1976, p.348.trad. C.Roels

21.Martin Heidegger, Etre et Temps, p.487

22. Martin Heidegger, Etre et Temps, p.479

23. Martin Heidegger, Nietzsche II , Paris, Gallimard,197, p.396.- trad. P. Klossowiski

24. Martin Heidegger, Lettre sur l'humanisme in Questions III, Paris, Gallimard, 1966, p.

83.- trad. R. Munier

25. Ibid, p.75.

26. Ibid, p.83.

27. Martin Heidegger, Acheminement vers la parole, Paris, Gallimard,1994, p.22.- trad. J. Beaufret

28. Martin Heidegger, Le principe de raison, p.95

29. Ibid, pp.95-96.

30. Martin Heidegger, Chemins qui ne mènent nulle part, Paris, Gallimard, 1962, p.105.trad. Wolfgang Brokmeir

31. Martin Heidegger, Essais et Conférences, p.9

32. Ibid, p.9.

33. Ibid, p.19.

34. Ibid, p.25.

35. Ibid, p.25.

36. Martin Heidegger, Questions I, Paris,Gallimard,1968, p.83.- trad. H.Corbin 
37. Martin Heidegger, Sérénité, in : Questions III, Paris, Gallimard, 1966, p.147.- trad. A. Préau

38. Martin Heidegger, Introduction à la philosophie, p.56.

39. Ibid, p.56.

40. Ibid, p.56.

41. Ibid, pp.48-49.

42. Martin Heidegger, Achèvement de la métaphysique et poésie, Paris, Gallimard, 2005, p. 167.- trad. A. Froidecourt,

43. Hölderlin, cité par Heidegger in Approche de Hölderlin, X , Paris, Gallimard, 1962, p. 113.- trad .C. Röels

44. Jean-Luc Marion, Dieu sans l'être, Paris, P.U.F, 2002.- p.42

45. Augustin Dibi, Préface in Pierre Ouattara, Quel chemin vers une patrie en Afrique?, Abidjan, éd. UCAO,2006

\section{RÉSUMÉS}

Si la question de l'être a été la préoccupation essentielle de Martin Heidegger, c'est pour qu'audelà de la détermination strictement philosophique, l'homme saisisse radicalement son essence pour y conformer son vécu quotidien dans ses aspirations les plus légitimes, parmi lesquelles nous avons la relation avec l'autre, comme valeur participant à la définition du je. A ce titre une pensée du mépris, de l'exclusion, du crime et de l'extermination ne saurait trouver un fondement dans la pensée de Martin Heidegger ; contrairement à ce que pense Emmanuel Faye.

If the question of to be, had been the preoccupation of Martin Heidegger, it's to invite humanity to radically think his essence for best comprehension, and take human as important reality. So that, it's not possible for Heidegger to think extermination, as thinking Emmanuel Faye.

\section{AUTEUR}

\section{JEAN GOBERT TANOH}

Dr Jean Gobert Tanoh, Maître-assistant et sous-directeur du département de philosophie de l' Université de Bouaké (Côte d'Ivoire) a notamment publié : «Heidegger contre Hegel», Koré, Université de Cocody, 2003 ; «L'appel à la raison comme appel de l'être», Repères, Université de Bouaké, 2004 ; «Concept vivant et choix: horizon d'une possibilisation de l'être africain», Ethiopiques $n^{\circ} 76$ (centenaire de Senghor), 2006 ; «Etre africain: approche métaphysique», Le Portique, e-Portique 2, 2006 ; rééd. in : Revue Géopolitique Africaine, $\mathrm{n}^{\circ}$ 27, 2007 ; «Concept et humanisme», Contrepoint philosophique, 2007. A paraître : De la vérité vivante du concept de développement, Conférence de Taipei en Taïwan, 2007. Ouvrages en préparation : De la mystique de la pensée à la mystique de Dieu, Le concept de vie dans le concept. tanohgobert@yahoo.fr 